\title{
CONFLICT OF INTEREST COMPLIANCE ARTICLE 1
}

As is standard in scholarly publishing, NCSBN's Journal of Nursing Regulation (JNR) requires its authors to disclose any potential conflicts of interest (COI). Although COI information has always been collected by our staff in order to support editors' review of the paper, it was not our standard practice to publish COI statements in each article. In this issue, JNR is retrospectively publishing the COI statements, which were collected with the below papers at submission, in order to make potential COl's transparent to readers, as well as editors.

1. "The 2017 National Nursing Workforce Survey" [Journal of Nursing Regulation, 2018;9(3S):S1-S88] https://doi.org/10.1016/ S2155-8256(18)30131-5

Declaration of competing interest: The authors declare that they have no known competing financial interests or personal relationships that could have appeared to influence the work reported in this paper.

2. "The Texas Board of Nursing Responds to Disaster" [Journal of Nursing Regulation, 2018;9(2):41-46] https://doi.org/10.1016/ S2155-8256(18)30116-9

Declaration of competing interest: The authors declare that they hav no known competing financial interests or personal relationships that could have appeared to influence the work reported in this paper.

3. "Advancing New Nurse Graduate Education Through Implementatio of Statewide, Standardized Nurse Residency Programs" [Journal of Nursing Regulation, 2018;8(4):14-21] https://doi.org/10.1016/ S2155-8256(17)30177-1

Declaration of competing interest: The authors declare that they have no known competing financial interests or personal relationships that could have appeared to influence the work reported in this paper.

4. "Board of Nursing Approval of Registered Nurse Education Programs" [Journal of Nursing Regulation, 2018;8(4):22-31] https://doi.org/10.1016/ S2155-8256(17)30178-3

Declaration of competing interest: The authors declare that they have no known competing financial interests or personal relationships that could have appeared to influence the work reported in this paper.

5. "2017 NCSBN Board of Nursing Website Audit" [Journal of Nursing Regulation, 2018;9(2):47-53] https://doi.org/10.1016/ S2155-8256(18)30117-0

Declaration of competing interest: The authors declare that they have no known competing financial interests or personal relationships that could have appeared to influence the work reported in this paper.
6. "The Difference Between Competency and Competence: A Regulatory Perspective" [Journal of Nursing Regulation, 2018:9(2):54-59] https://doi.org/10.1016/S2155-8256(18)30118-2

htps://doi.org/10.1016/S2155-8256(18)30118-2 Declaration of competing interest: The authors declare that they have no known competing financial interests or personal relationships that
could have appeared to influence the work reported in this paper.

7. "Reporting of Nurse Discipline to the National Practitioner Data Bank" [Journal of Nursing Regulation, 2018;9(2):31-40] https://doi.org/10.1016/ 2155-8256(18)30115-7

Declaration of competing interest: The authors declare that they hav no known competing financial interests or personal relationships that could have appeared to influence the work reported in this paper.

8. “Comparing Nurse Practitioner Student Diagnostic Reasoning Outcomes in Telehealth and Face-to-Face Standardized Patient Encounters" [Journal of Nursing Regulation, 2018;9(3):27-35] https://doi. org/10.1016/S2155-8256(18)30151-0

Declaration of competing interest: The authors declare that they have no known competing financial interests or personal relationships that could have appeared to influence the work reported in this paper.

9. "Patient Safety Culture and Barriers to Adverse Event Reporting: A National Survey of Nurse Executives" [Journal of Nursing Regulation, 2018;9(2):9-17] https://doi.org/10.1016/S2155-8256(18)30113-3 Declaration of competing interest: The authors declare that they have no known competing financial interests or personal relationships that could have appeared to influence the work reported in this paper.

10. "Bibliometrics: A Means of Visualizing Occupational Licensure Scholarship" [Journal of Nursing Regulation, 2018;9(1):31-37] https://doi. org/10.1016/S2155-8256(18)30052-8

Declaration of competing interest: The authors declare that they have no known competing financial interests or personal relationships that could have appeared to influence the work reported in this paper.
11. "Qualitative Research in Nursing and Health Professions Regulation" [Journal of Nursing Regulation, 2018;9(3):15-26] https://doi.org/10.1016/ S2155-8256(18)30150-9

Declaration of competing interest: The authors declare that they have no known competing financial interests or personal relationships that could have appeared to influence the work reported in this paper.

12. "Investigating the Relationship Between Continuing Competence and Insight in Nursing and Midwifery Practice" [Journal of Nursing Regulation, 2018;9(3):36-52] https://doi.org/10.1016/S2155-8256(18)30152-2 Declaration of competing interest: The authors declare that they have no known competing financial interests or personal relationships that could have appeared to influence the work reported in this paper.

13. “Instituting Evidence-Based Changes to Improve First-Time NCLEXRN ${ }^{8}$ Pass Rates" [Journal of Nursing Regulation, 2018;9(1):11-18] https://doi.org/10.1016/S2155-8256(18)30049-8

Declaration of competing interest: The authors were contacted after publication to request a Declaration of Interest statement.

14. "Delaware's Pathway to Achieving APRN Consensus" [Journal of Nursing Regulation, 2018:9(1):23-30] https://doi.org/10.1016/ S2155-8256(18)30051-6

Declaration of competing interest: The authors were contacted after public ation to request a Declaration of Interest statement.

15. “The APN-PLACE Telehealth Education Network: Legal and Regulatory Considerations" [Journal of Nursing Regulation, 2018:9(1):47-51] https//doi.org/10.1016/S2155-8256(18)30054-1

Declaration of competing interest: The authors were contacted after publication to request a Declaration of Interest statement. 